\title{
Bebês: o irresistível poder da graciosidade. Um estudo sobre o significado evolutivo dos traços infantis
}

\author{
Monique Leitão \\ Instituto Federal de Educação, Ciência e Tecnologia do Rio Grande do Norte \\ Rochele Castelo-Branco \\ Universidade Federal do Rio Grande do Norte
}

\begin{abstract}
Resumo
Os bebês, em geral, despertam ternura e comportamentos de cuidado nas pessoas. O objetivo deste trabalho foi discutir a atração provocada pelos traços infantis, tendo como referencial teórico os quatro níveis de explicação de Tinbergen. Do ponto de vista dos mecanismos que desencadeiam essa atração neonatal, avaliamos a importância da aparência do infante para eliciar as respostas de cuidado parental. Considerando a função adaptativa desta característica, discutimos os processos evolutivos que moldaram as preferências pelos atributos infantis, além da história filogenética do favoritismo pelos sinais morfológicos de filhotes. Apresentamos, ainda, dados sobre o desenvolvimento deste traço comportamental ao longo da vida. Tomadas em conjunto, estas informações provêem uma análise de por que achamos os bebês tão irresistíveis.
\end{abstract}

Palavras-chave: bebê; cuidado parental; fofura; baby schema.

\begin{abstract}
Babies: the irresistible power of cuteness. A study concerning the evolutionary function of infantile traits. Babies generally elicit tenderness and caretaking behaviors on people. The aim of this study is to discuss the attraction towards babyish features, taking Tinbergen's four questions as a theoretical benchmark. Concerning the mechanisms implicated in this neonatal attraction, we evaluated the importance of children's morphology on eliciting parental responses. Considering the adaptive function of this characteristic, we discuss the evolutionary process that shaped the preferences towards infantile traits as well as the phylogeny of the preference towards infant's morphological traits. It was also presented data about the development of this behavioral trait across life span. The information provided here suggests a reason why we find babies such irresistible creatures.
\end{abstract}

Keywords: baby; parental caretaking behavior; cuteness; baby schema.

$\mathrm{V}$ ocê já se perguntou por que os bebês são tão graciosos? Por que eles parecem tão adoráveis a ponto de nos desarmar diante de seus caprichos? Sem dúvida, os bebês humanos são capazes de despertar sentimentos afetuosos, de tal modo que, misteriosamente, nos sentimos convidados a interagir com eles, a nos aproximar e, até mesmo, a dirigir carinhos e cuidados a estes pequenos. Na realidade, os bebês nos parecem amáveis porque estamos tanto culturalmente como biologicamente preparados para assim os ver, o que desperta em nós mesmos posturas também amáveis em direção a eles. Neste artigo, nosso objetivo será discutir por que nos sentimos tão atraídos pelos bebês ou pelos chamados "atributos infantis", buscando articular conhecimentos teóricos construídos na literatura às mais recentes pesquisas que investigam esta temática.

Para guiar a discussão sobre os diversos fatores que estão relacionados aos sentimentos afetivos direcionados aos infantes, tomaremos como base as quatro questões propostas pelo etólogo Nikolaas Tinbergen para o estudo do comportamento. Segundo Tinbergen (1963/2005), existiriam quatro perspectivas diferentes para compreender e estudar o comportamento, as quais se remetem a explicações independentes, mas complementares (Alcock, 2001; Izar, 2009). Assim, as quatro questões se referem a: 1) Mecanismos causais do comportamento, ou seja, mecanismos fisiológicos (neurais, sensoriais, hormonais e motores) envolvidos no comportamento, bem como estímulos que o desencadeia; 2) Desenvolvimento, isto é, a origem ontogenética do comportamento e fatores ambientais envolvidos; 3) Função adaptativa, que diz respeito ao valor do comportamento para a sobrevivência e reprodução; e 4) História evolutiva, que faz referência a como o comportamento se desenvolveu na espécie estudada e por que ele seguiu certo processo evolutivo.

O presente artigo está estruturado em quatro grandes blocos, cada um dos quais referentes aos níveis de análises distintos, 
como proposto por Tinbergen. Assim, a nossa pergunta base, "Por que nos sentimos tão atraídos pelos traços infantis?", será discutida ao longo do texto a partir de questões desmembradas, com o objetivo de abranger as causas próximas e últimas envolvidas neste tema, conforme indica a figura 1.

\section{Mecanismos naturalmente encantadores}

O esquema infantil. Um dos primeiros estudiosos

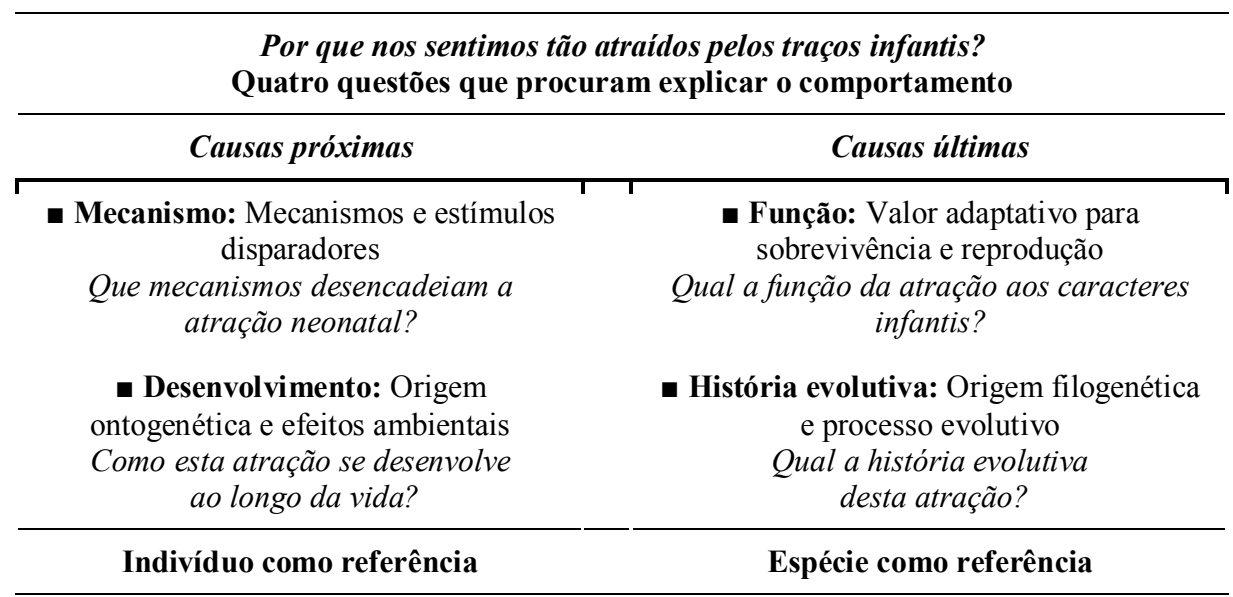

Figura 1. Descrição das quatro questões de Tinbergen que fundamentam a discussão sobre a atratividade a caracteres infantis neste artigo

que buscou compreender os mecanismos que desencadeiam as respostas afáveis aos bebês foi o etólogo austríaco Konrad Lorenz. Em 1943, Lorenz propôs que certos atributos presentes nos bebês, os chamados "esquemas de aspectos infantis" ou "esquemas de bebê" (do alemão kindchenschema ou do inglês baby schema), seriam capazes de desencadear respostas emocionais de afeto e cuidado nos adultos (Lorenz, 1971). Para Lorenz, os bebês humanos, quando comparados ao padrão adulto, apresentam um conjunto de características particulares: olhos grandes em relação ao rosto e de implantação baixa, cabeça arredondada e grande em relação ao corpo, testa protuberante e grande em relação ao rosto, queixo retraído, nariz pequeno, bochechas mais arredondadas e salientes, corpo relativamente pequeno, membros curtos e rechonchudos (Figura 2). Estes atributos físicos, combinados a alguns comportamentos, como os movimentos desajeitados e o sorriso, fazem com que os bebês sejam vistos de forma extremamente afetuosa (Hrdy, 2001; Lorenz, 1971; Lorenz, 1981/1995).

Lorenz (1981/1995) considerou as respostas de ternura direcionadas aos bebês um bom exemplo do efeito de um Mecanismo Liberador Inato (MLI). Abandonando o termo esquema, que fora cunhado anteriormente, o autor passa a usar a expressão MLI para referir-se à organização neural envolvida na habilidade de identificar e responder de modo adequado, e sem experiência anterior, a situações biologicamente relevantes. Neste sentido, os traços tipicamente infantis mencionados por Lorenz funcionariam como estímulos-chave para desencadear um mecanismo inato liberador ou disparador de emoções e comportamentos de cuidado parental (Lorenz, 1981/1995).

Diversos estudos têm mostrado a relação entre os traços do esquema de bebê e maiores níveis de percepção de fofura, jovialidade, atratividade, preferência ou atenção, o que confirma experimentalmente a teoria de Lorenz. Os bebês que apresentam faces mais próximas ao padrão infantil (naturalmente ou experimentalmente manipuladas), por exemplo, são também percebidos como mais fofinhos (Glocker et al., 2009a; Hildebrandt \& Fitzgerald, 1979). Além disso, a alteração de apenas um traço específico é capaz de afetar a percepção dos bebês. Manipulações em desenhos de crianças mostraram que quanto mais o formato da cabeça seguia o esquema infantil, mais os desenhos eram avaliados como fofinhos (Alley, 1981).

A aparência do bebê parece informar também sobre a idade do mesmo. Bebês que seguem o esquema de caracteres infantis

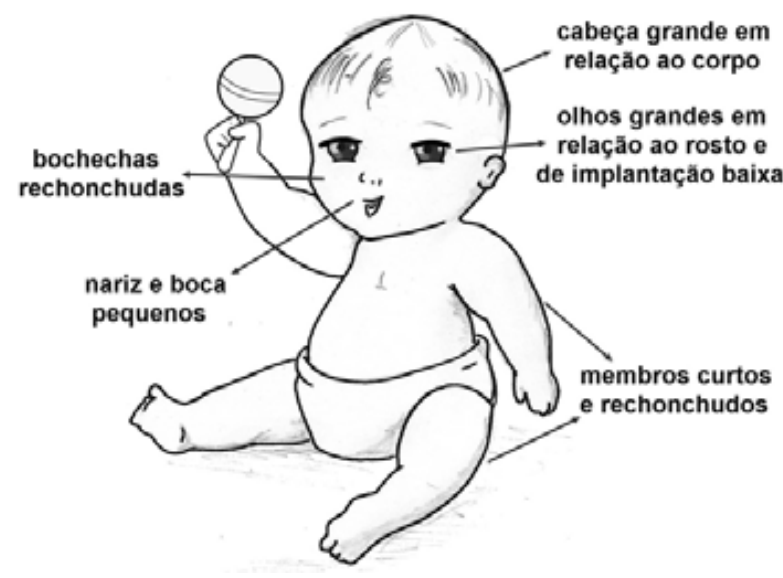

Figura 2. Representação de um bebê, destacando as características infantis típicas. 
são julgados como mais fofinhos e também são vistos como mais novos (Lobmaier, Sprengelmeyer, Wiffen, \& Perrett, 2010). Do mesmo modo, bebês avaliados como menos atrativos são considerados mais velhos, tendo suas habilidades superestimadas para sua idade, ainda que sejam vistos como tendo competência geral mais baixa do que bebês atrativos (Ritter, Casey, \& Langlois, 1991). Em outro trabalho, constatou-se entre adultos um padrão de maior preferência por faces infantis a faces adultas, tanto em imagens de humanos como de não-humanos, o que mostra que a preferência pelo esquema infantil se estende para faces de outros animais (Fullard \& Reiling, 1976).

$\mathrm{Na}$ verdade, a afeição provocada pelos traços infantis nos bebês se estende a qualquer elemento que apresente estes traços. É por isso que os filhotes de outros mamíferos, algumas personagens de desenho animado, bonecos ou ursinhos de pelúcia nos parecem tão atraentes.

Em relação aos ursinhos de pelúcia, por exemplo, a indústria de brinquedos os deixou cada vez mais parecidos com o esquema de bebê, trocando o focinho e membros alongados típicos de um urso adulto por um focinho curto e membros rechonchudos (Hinde \& Barden, 1985). O mesmo processo sofreu o famoso Mickey Mouse, que ao completar 50 anos ganhou uma homenagem do cientista Stephen Jay Gould, em um artigo que trata sobre as alterações em seu desenho. Gould (1989) mostrou que, ao longo dos anos, o camundongo tornou-se mais bem-comportado e seus traços ganharam o toque infantil, como olhos maiores, cabeça maior em relação ao corpo e membros mais grossos. O que chama atenção é que Mickey, apesar de ser um adulto, permanece representado como eternamente jovem. Esta manutenção das características juvenis, comportamentais ou anatômicas, durante a infância até a vida adulta de um animal convencionou-se chamar de neotenia (Gould, 1989). Sob a perspectiva filogenética, a neotenia é definida pela presença de características em indivíduos adultos de uma determinada espécie que se assemelham a características de indivíduos jovens de espécies próximas ou ancestrais (Futuyma, 1992).

No mundo animal, é possível encontrar alguns exemplos de neotenia. Entre muitos animais de estimação é perceptível a retenção de traços infantis nos indivíduos adultos, o que ajuda a promover o sentimento de amabilidade que temos em relação aos mesmos (Archer, 1997). Algumas raças de cachorro, como por exemplo, o Maltês e o Yorkshire Terrier, parecem ter sofrido um processo de seleção artificial para o favorecimento da neotenia.

Mecanismos neurais e hormonais. Segundo as proposições de Lorenz (1981/1995), a idéia de que os humanos reagem perceptual e comportamentalmente à estrutura da face dos bebês está diretamente ligada à existência de uma organização neural que apreende e decodifica tais estímulos. Muitos anos após estas primeiras teorizações, a ciência desenvolveu tecnologias suficientes para testar e indicar a base neurológica para o MLI de Lorenz.

Utilizando-se de técnicas modernas de neuroimagem funcional, pesquisadores identificaram um padrão específico de atividade neural exibido apenas enquanto indivíduos visualizam faces de crianças (Kringelbach et al., 2008). Neste estudo, constatou-se que as imagens dos bebês provocavam a imediata ativação do córtex órbito-frontal medial dos sujeitos, uma área localizada no sistema límbico (região do cérebro responsável pela produção das emoções). Recentemente, outro experimento avaliou as respostas neurais evocadas em adultos durante a visualização de faces manipuladas de bebês, tendo verificado uma correlação entre a intensidade do esquema de aspectos infantis das imagens e a ativação de uma outra estrutura do sistema límbico, o nucleus accumbens (Glocker et at., 2009b). Este dado reforça a concepção de que o envolvimento de mecanismos neurais motivacionais é a base biológica para a resposta afetiva aos bebês.

Além de mecanismos neurais, mecanismos hormonais também estão intimamente envolvidos na atração neonatal, especialmente hormônios femininos. Em uma recente pesquisa, mulheres jovens que usavam contraceptivos orais foram mais sensíveis em perceber alterações nos traços de fofura em bebês do que mulheres que não utilizam contraceptivos orais (Sprengelmeyer et al., 2009). Neste mesmo estudo, mulheres que já haviam passado pela menopausa (que diminui as taxas de progesterona e estrógeno) apresentaram-se menos discriminativas em relação às alterações de fofura em imagens de bebês do que mulheres da mesma faixa etária que não tinham passado pela menopausa.

Analisadas em conjunto, as pesquisas e proposições teóricas levantadas nesta primeira parte deste artigo nos permitem compreender melhor a gama de mecanismos envolvidos na atração neonatal. Tais estudos confirmam experimentalmente os elementos que compõem um MLI, uma vez que indicam a existência de liberadores (estímulos específicos presentes na face dos bebês ou de outros animais/objetos) e efeitos de liberação (estrutura neural que reage ao liberador e elicia a resposta comportamental, modulada por mecanismos hormonais).

\section{Necessidade de cuidado: a fragilidade do bebê humano}

Para entender melhor a importância evolutiva dos caracteres infantis, cabe ressaltar a fragilidade do recém-nascido humano. Em relação a outros primatas, nossos bebês são extremamente dependentes e necessitam de cuidados bem mais prolongados (Silk, 1999). Nascemos muito indefesos e impotentes, assim, somos incapazes de sobreviver se não contarmos com adultos para nos fornecer alimentação, nos locomover e prover proteção contra predadores (Vieira \& Prado, 2004).

Na verdade, os bebês humanos nascem em um período de desenvolvimento muito prematuro, pois, fazendo um paralelo com o tempo gestacional dos demais primatas hominóides (gorilas, orangutangos, bonobos e chimpanzés, dentre outras espécies com as quais estamos mais aparentados), a gestação humana deveria ter 12 meses a mais do que os nove meses existentes (De Toni, De Salvo, Marins, \& Weber, 2004; Leakey, 1997). Esta antecipação do nascimento do bebê humano está relacionada às pressões seletivas que levaram à postura bípede no gênero Homo e que tiveram como conseqüência o estreitamento do quadril e do canal pélvico. $O$ reduzido canal de passagem impossibilitava o parto de neonatos maiores, o que levou à antecipação do período gestacional (Rosenberg \& Trevathan, 1996). Em virtude disso, comparado aos demais primatas, o recém-nascido humano é mais imaturo fisicamente, tanto no 
aspecto motor como perceptivo (Bjorklund, 1997; Hrdy, 2001).

Além da acentuada imaturidade do neonato, nossa espécie tem como forte característica o longo período de desenvolvimento. Temos o maior período de infância e somos os únicos que mantemos o cuidado à alimentação dos filhos mesmo durante a adolescência (Bjorklund, 1997). A longa imaturidade dos Homo sapiens pode ser conseqüência da seleção para a grande capacidade de aprendizado e está relacionada ao tempo necessário para a apreensão das regras e papéis sociais e para a aquisição da herança cultural que construímos em gerações anteriores (Futuyma, 1992; Vieira \& Prado, 2004).

Para viabilizar a sobrevivência de uma prole tão dependente por tanto tempo, foi preciso haver alto nível de investimento parental. Deste modo, pressões seletivas favoreceram nos humanos o forte vínculo mãe-bebê, o desenvolvimento do apego, a presença do macho e a formação da família (em suas diversas configurações), bem como a participação de outros adultos na assistência aos infantes (Bowlby, 1969/1990; De Toni et al., 2004; Eibl-Eibesfeldt, 1989; Hrdy, 2001).

Diante deste cenário, o bebê humano também precisou desenvolver armas poderosas para evocar a atração e a interação com os adultos, dentre estas, a capacidade de imitar expressões faciais ao nascer (Meltzoff \& Moore, 1977), a atenção à fala e à face humana (Brody, Zelazo, \& Chaika, 1984; Fantz, 1963) e, especialmente, o esquema de atributos físicos e comportamentais infantis. Todos estes artifícios encantadores têm como função adaptativa promover a sobrevivência do bebê, uma vez que eliciam cuidados essenciais a este, desde a alimentação à proteção e conforto, podendo, inclusive, diminuir o risco de abandono.

Uma outra característica interessante a ser destacada em bebês humanos é a quantidade de gordura que eles apresentam. Surpreendemente, os recém-nascidos de nossa espécie têm de quatro a oito vezes mais gordura corporal do que um bebê de macaco, o que representa $16 \%$ de seu peso (Hrdy, 2001). Dentre tantas teorias para explicar este fenômeno - como a de isolamento térmico, de reserva energética e de recursos para a custosa atividade cerebral - uma teoria em particular defende que a gordura corporal funcionaria como uma "autopublicidade" para o bebê, anunciando a sua capacidade de sobreviver (Hrdy, 2001). De fato, o peso do neonato é uma razoável medida prognóstica para a sobrevivência, saúde e desenvolvimento mental posterior, o que dá força a esta hipótese. Segundo esta idéia, a fofura do bebê, exibida em suas formas rechonchudas, estaria informando seu potencial em sobreviver. Algumas tentativas anteriores de explicar as bochechas gorduchas dos bebês, por exemplo, defendiam uma suposta participação na sucção, o que não se sustenta, dada a falta deste atributo em outros primatas (Lorenz, 1981/1995).

É importante ressaltar que a atração provocada pelo bebê não se direciona apenas aos pais biológicos, pois, na verdade, esta tem como alvo qualquer cuidador em potencial. Nos humanos e em certos mamíferos, a ajuda de outros membros do grupo se mostra primordial no cuidado dos filhotes. Mitani e Watts (1997) mostraram que mães primatas de várias espécies que contam com boa assistência no cuidado aos seus filhotes apresentam gestações em intervalos mais curtos e seus filhotes crescem mais rápido do que mães que cuidam sozinhas de sua prole. Estes ajudantes que são chamados de "alomães", são geralmente fêmeas subordinadas e fêmeas imaturas reprodutivamente e, especialmente no caso dos humanos, temos ainda os avós da criança (parentes fora da idade reprodutiva) (Hrdy, 2001).

Diversos estudos experimentais apontam para a relação entre algumas características dos infantes e a disposição dos pais ao cuidado. Em um desses estudos, crianças que foram avaliadas como mais fofinhas, receberam mais sorrisos e vocalizações de seus pais (Hildebrandt \& Fitzgerald, 1983), de modo semelhante, crianças que apresentavam faces menos infantilizadas eliciaram menos cuidados e tenderam a inibir menos eficazmente a agressão por adultos (Frodi et al., 1978; Maier, Holmes, Slaymaker, \& Reich, 1984). Um recente estudo mostrou que faces de bebês altamente infantilizadas não só foram avaliadas como mais fofinhas, como também eliciaram forte motivação para o cuidado quando comparadas a faces pouco infantilizadas (Glocker et al., 2009a).

Estes resultados confirmam as hipóteses previamente descritas de que os traços infantis induzem o comportamento de cuidado, tendo como função biológica assegurar a sobrevivência do bebê. Nesse sentido, os bebês que são capazes de melhor anunciar seus caracteres juvenis garantem que serão percebidos como graciosos, conquistando cuidados e carinhos por parte de adultos.

\section{Era uma vez... um filhote que foi escolhido pelos seus} pais

Do ponto de vista filogenético, os seres humanos não são os únicos a apresentar preferências a atributos infantis. Para entender a história evolutiva desta atratividade, é preciso compreender que esta ocorre particularmente entre espécies que possuem alto investimento parental, como nos mamíferos e aves, em geral. Nestes animais, os pais costumam ocupar uma posição de exercer favoritismo ou escolha parental em relação à prole, que compete entre si para chamar a atenção dos pais (West-Eberhard, 1983).

Entre as aves, filhotes de certas espécies apresentam ornamentos que somente estão presentes durante o período de dependência parental, especialmente quando apenas os pais alimentam os filhotes, o que sugere que tais ornamentos têm um importante papel na competição por cuidado (Krebs \& Putland, 2004). A plumagem vistosa e brilhante dos neonatos de frangosd'água americanos (Fulica americana), por exemplo, está intimamente relacionada à escolha parental. Um estudo pioneiro mostrou que filhotes ornamentados receberam mais alimento, cresceram mais e tiveram maior taxa de sobrevivência do que os experimentalmente não ornamentados (Lyon, Eadie, \& Hamilton, 1994). Reconstruções filogenéticas da família Rallidae (que inclui os frangos-d'água americanos) sugerem que o padrão não ornamentado era o estado ancestral e que ao longo de gerações, o favoritismo parental em algumas espécies resultou em aumento da aptidão da prole com plumagem exuberante, promovendo a seleção destes traços nos imaturos.

Em muitas espécies de primatas, os filhotes apresentam a pele ou os pêlos coloridos, os quais mudam de cor ao longo da vida e possuem a função de atrair o cuidado de adultos (Gerald, 
Waitt, \& Maestripieri, 2006). Em uma extensa revisão de literatura, Alley (1980) assinala várias evidências que apontam para a significativa função da coloração infantil entre várias espécies de macacos do velho mundo (Cercopithecidae) e entre grandes macacos (Pongidae). Segundo este autor, dados sobre a ecologia de primatas, além de observações de indivíduos em campo ou cativeiro, indicam que a coloração da pelagem de filhotes pequenos pode estar relacionada à atenção, proteção, tolerância e cuidado por parte de conspecíficos, sendo utilizada para distinguir filhotes dependentes daqueles que já são mais velhos e mais independentes (Treves, 1997). Em macacos rhesus (Macaca mulatta), por exemplo, um estudo aponta que fêmeas passam mais tempo com filhotes com faces cor-de-rosa, que é o padrão do recém-nascido nos primeiros dias de vida (Higley, Hopkins, Hirsch, Marra, \& Suomi, 1987).

Na espécie humana, não seria tão diferente. Sarah Hrdy (2001) defende que os traços infantis teriam evoluído como uma atração neonatal, sugerindo que a preferência de adultos por certos caracteres infantis tenha favorecido a intensificação e manutenção dos famosos traços, já mencionados por Lorenz (1981/1995). De fato, estes atributos permanecem por mais tempo no desenvolvimento humano do que no de outros mamíferos, parecendo anunciar a jovialidade aos nossos cuidadores.

É interessante observar que, se por um lado, o poder da seleção sexual na evolução de atributos de machos e fêmeas seja amplamente conhecido na literatura (Buss, 2003; Fisher, 1915), pouca atenção tem sido dada à força da escolha parental na seleção desenfreada por traços infantis. Ao longo de gerações, o tratamento preferencial dos pais a filhotes que possuíam algum atributo particular promoveu a exacerbação deste atributo, uma vez que aqueles que o possuíam foram mais bem alimentados ou bem protegidos, o que conseqüentemente, aumentou a aptidão de tais filhotes favorecidos (West-Eberhard, 1983).

Deste modo, compreende-se que a história evolutiva dos atributos infantis tenha se iniciado de forma muito modesta, mas que com o tempo gerou traços exuberantes entre diferentes filhotes de animais, uma vez que ao mesmo tempo favorecia filhotes vistosos e pais que possuíam preferência para isso. Através da escolha parental, qualquer traço que gere atratividade dos pais é selecionado por um processo desenfreado, no qual o referido traço será favorecido pelos pais nos filhotes, que, por sua vez, se tornarão adultos extremamente atraídos pelo mesmo traço em seus descendentes.

\section{A origem da afeição a bebês}

Em uma primeira análise, pensar sobre a atração a caracteres infantis nos remeteria primordialmente à expressão desta preferência nos adultos, ou seja, nos cuidadores, mas não naqueles que recebem os cuidados, que seriam as crianças ou os bebês. No entanto, como já comentamos anteriormente, é evidente a grande aceitação alcançada entre o público infantil no que se refere aos brinquedos que simulam as características descritas por Lorenz em seu baby schema. Com base no exposto, poderíamos nos perguntar: em que período da vida surge esta atração por atributos infantis?

Na realidade, perceber e reagir a feições são comportamentos que se apresentam já em neonatos, dada a importância da leitura de expressões faciais na interação social humana (Fantz, 1963; Meltzoff \& Moore, 1977; Mondloch et al., 1999). Além disso, a responsividade de crianças a faces consideradas esteticamente belas segue as mesmas tendências observadas em adultos (Langlois et al., 1987; Langlois, Ritter, Roggman, \& Vaughn, 1991; Quinn, Kelly, Lee, Pascalis, \& Slater, 2008).

Sabendo que crianças são capazes de reagir de modo particular a estímulos da face, resta-nos descobrir se elas são sensíveis a características que denotam fofura. Um dos primeiros estudos, neste campo, comparou crianças e adolescentes quanto à preferência por imagens de infantes ou de adultos e mostrou que crianças entre aproximadamente sete e doze anos preferiram imagens de rostos de adultos a imagens infantis, contudo, entre adolescentes houve preferência pelas imagens infantis, tendo inclusive as meninas apresentado preferência mais acentuada $\mathrm{e}$ mais precoce do que os meninos (Fullard \& Reiling, 1976). Para os autores, a preferência por infantes iniciaria na adolescência porque, nesta fase, os jovens se tornam reprodutivamente maduros e aptos a exercer o papel de pais.

Por outro lado, o já mencionado rejuvenescimento das feições do Mickey Mouse (Gould, 1989), bem como o processo de infantilização dos integrantes brasileiros da Turma da Mônica, parecem evidenciar uma acomodação dos desenhos aos gostos infantis. Além disso, é interessante notar que crianças mais velhas já apresentam alguns comportamentos típicos da interação com os pequenos. Crianças com menos de 6 anos, por exemplo, quando em contato com bebês, levantam as sobrancelhas, afinam a voz e falam em "mamanhês" ou a chamada fala dirigida a bebês (aquele jeito gracioso de comunicação característico das mamães com os seus bebês), tal qual ocorre com adultos (Zebrowitz, 1997).

Em um estudo com ursinhos de pelúcia, Morris, Reddy e Bunting (1995), apontam uma faixa etária específica na qual a preferência por caracteres infantis parece começar a se expressar. Nesta pesquisa, crianças na faixa dos 4 anos tenderam a eleger, como preferidos, ursinhos que lembravam o animal adulto. Entretanto, crianças de 6 e 8 anos escolheram os ursos com formas parecidas com as de um filhote. Ademais, foi possível verificar maior interesse por exercer comportamentos de cuidado ao urso favorito em crianças mais velhas, pois a preferência por dormir ou abraçar este brinquedo aumentaram com a idade.

Do ponto de vista da ontogenia, percebemos, então, que existem predisposições na infância para responder diferencialmente a outras crianças. Será que estas informações seriam suficientes para anunciar o caráter precoce do MLI de Lorenz? Com o intuito de constatar experimentalmente a preferência específica das crianças pelo baby schema, Sanefuji, Ohgami e Hashiya (2007) testaram adultos e crianças de 5 anos de idade em relação a fotografias de animais (inclusive o homem) de diversas faixas etárias. A preferência pelos animais em suas primeiras etapas de desenvolvimento foi unânime entre os sujeitos. Mesmo aos cinco anos, as crianças apresentaram respostas similares aos adultos quanto ao esquema infantil, o que aponta para uma precoce expressão do poder dos traços de jovialidade.

Uma análise conjunta dos resultados descritos acima sugere que a atração aos caracteres infantis pode emergir cedo na 
infância. Entretanto, ainda é preciso investigar esta preferência em crianças de diferentes idades para estabelecer quando exatamente esta começa a se expressar na escala ontogenética. Resultados preliminares de um recente estudo apontam que crianças entre 9 e 10 anos são mais sensíveis do que as de 3 e 4 anos a traços de infantilidade em imagens experimentalmente manipuladas (Castelo-Branco, Leitão, Dantas, Lopes, \& Yamamoto, 2009). É fácil entendermos esses dados quando consideramos a função evolutiva do envolvimento infantil no cuidado de irmãos mais novos ou de bebês não aparentados, comportamento comum em algumas sociedades (Cole \& Cole, 1989/2003).

Certamente, o desenvolvimento da atração por caracteres infantis também é modulado por fatores sociais e culturais ao longo da vida do indivíduo. O relacionamento entre irmãos durante a infância ilustra a influência desses aspectos. Em contextos familiares nos quais ambos os progenitores trabalham, como em sociedades indígenas, comunidades agrárias, famílias de baixo poder aquisitivo ou famílias de imigrantes, o cuidado de crianças aos irmãos mais novos é uma prática comum (Dahlblom, Herrara, Peña, \& Dahlgren, 2009; Hafford, no prelo; Poletto, Wagner, \& Koller, 2004). Alimentar, observar, ler histórias infantis, intermediar as brincadeiras, preparar e acompanhar os irmãos mais novos à escola são algumas das atividades que os pais delegam aos filhos.

Entre os !Kung San, grupo de caçadores-coletores que vivem no deserto do Kalahari, os bebês, ao atingirem um ano de idade, passam a ser cuidados por grupos de crianças para que a mãe retorne ao trabalho de coleta de alimentos (Konner, 1977). Similarmente, nos Gusii, comunidade agrária que vive no Quênia, boa parte dos cuidados às crianças é proferida por seus irmãos ou tias jovens, podendo haver apenas quatro anos de diferença entre a idade dos cuidadores e dos que são cuidados (Zukow-Goldring, 1995).

Nesse contexto, pesquisas que investigaram crianças a respeito do cuidado dispensado aos irmãos mais novos demonstraram que a experiência é geralmente avaliada como positiva por partes dos jovens cuidadores (Dahlblom et al., 2009; Kosonen, 1996). É de se esperar, assim, que no âmbito dessas atividades, o apego e atração aos bebês sejam socialmente estimulados.

É possível perceber, também, que a cultura de um povo que inclui crenças, valores, tradições e normas sociais - constrói diferentes concepções sobre quem são os bebês, influenciando diretamente a forma de interagir com os mesmos. Os Kalulis, originários de Papua Nova Guiné, por exemplo, acreditam que os bebês são seres sem entendimento e também consideram inadequado olhar para a pessoa ao falar com ela, assim, as mães kalulis não se envolvem em contato visual prolongado com os bebês e os seguram sem olhar para eles (Ochs \& Scieffelin, 1984). Já entre as mães ocidentais, como as norte-americanas, por exemplo, são freqüentes o contato visual e as interações entusiasmadas com seus pequenos (Brazelton, Nugent, \& Lester, 1974). É provável que comportamentos e crenças culturais, como as citadas, influenciem a forma como as pessoas reagem e se sentem atraídas por traços infantis. Neste sentido, estudos ainda precisam ser desenvolvidos para que esta hipótese possa ser diretamente investigada.

Mesmo estando claro que a atratividade a infantes não é uma característica exclusiva da fase adulta, ainda há muito a ser compreendido a respeito do seu desenvolvimento ao longo da vida, especialmente sobre a influência da aprendizagem e atuação dos fatores sócio-culturais neste processo.

Integrando os traços do baby squema. As características infantis típicas, em especial a estrutura do rosto da criança, são mecanismos naturalmente encantadores capazes de desencadear emoções e comportamentos ternos em adultos. Neste processo, a configuração do aparato neural e hormonal humano contribui fortemente para a expressão da afeição por crianças. A atuação desses fatores, inclusive, ocorre desde muito cedo na vida dos indivíduos, sendo observados mesmo na infância. Do ponto de vista evolutivo, discutimos a relevância dos atributos infantis para motivar o investimento parental, uma vez que os bebês humanos são muito frágeis e imaturos, exigindo intensa assistência dos cuidadores. Ademais, o apelo exibido pelos atributos infantis tem a importante função de anunciar a potencialidade do infante em sobreviver, atuando positivamente em situações de ambivalência por parte da mãe. As preferências por caracteres infantis, inclusive, não são exclusivas da espécie humana, estando diretamente ligadas à condição de elevado investimento parental.

É importante enfatizar que a atratividade a traços infantis, como qualquer outra característica da espécie humana, deve ser compreendida sempre a partir dos múltiplos fatores envolvidos. $\mathrm{O}$ intuito deste trabalho foi reunir as mais diversas explicações sobre esta temática, pois todas elas podem ser elucidadas a partir da complementaridade das quatro questões de Tinbergen.

O presente trabalho, desse modo, oferece uma análise do valor dos traços infantis à luz da perspectiva evolucionista, procurando integrar diferentes peças deste grande quebracabeça, peças estas que, geralmente, encontram-se fragmentadas na literatura. Ainda que tenhamos contemplado os principais aspectos envolvidos nesse fenômeno, certamente existem muitos outros além dos aqui discutidos.

De modo geral, ao longo do texto, foram apresentados indícios das bases biológicas da atratividade a caracteres infantis, além da influência de fatores culturais na atração por infantes. Se por um lado, somos biologicamente predispostos a reagir aos bebês, é no âmbito das relações sociais que aprendemos a tratar e interagir com eles, percebendo-os como fofinhos e prestandolhes cuidados. Assim, aspectos culturais e biológicos caminham juntos para tornarem irresistíveis os traços infantis.

\section{Agradecimentos}

Agradecemos a Aline Araújo pela ilustração; e a Ana Cláudia Nunis e a Ana Ludmila Costa pelas contribuições ao manuscrito.

\section{Referências}

Alcock, J. (2001). Animal behavior: an evolutionary approach (5 $5^{\underline{a}}$ ed.). 
Sunderland: Sinauer Associates.

Alley, T. R. (1980). Infantile colouration as an elicitor of caretaking behaviour in old world primates. Primates, 21(3), 416-429.

Alley, T. R. (1981). Head shape and the perception of cuteness. Developmental Psychology, 17(5), 650-654.

Archer, J. (1997). Why do people love their pets? Evolution and Human Behavior, 18(4), 237-259.

Bjorklund, D. F. (1997). The role of immaturity in human development. Psychological Bulletin, 122(2), 153-169.

Bowlby, J. (1990). Apego e perda - Apego: a natureza do vínculo (Vol. 1) (2 $2^{\mathrm{a}}$ ed.) (A. Cabral, Trad.). São Paulo: Martins Fontes (Publicado originalmente em 1969)

Brazelton, T. B., Koslowski, B., \& Main, M. (1974). The origin of reciprocity: the early mother-infant interaction. In M. Lewis \& L. Rosenblum (Orgs.), The effect of the infant on its caretaker (pp. 133-157). New York: Wiley.

Brody, L. R., Zelazo, P. R., \& Chaika, H. (1984). Habituation-dishabituation to speech in the neonate. Developmental Psychology, 20(1), 114-119.

Buss, D. M. (2003). The evolution of desire: strategies of human mating. New York: Basic Books.

Castelo-Branco, R., Leitão, M., Dantas, C., Lopes, F. A., \& Yamamoto, M. E. (2009). Younger, then cuter: children prefer babish babies [CD ROM]. In Instituto do Milênio: Psicologia Evolucionista (Org.), Simpósio Internacional Psicologia Evolucionista no Milênio: plasticidade e adaptação. Natal: Autor.

Cole, M., \& Cole, S. R. (2003). O desenvolvimento da criança e do adolescente ( $4^{\underline{a}}$ ed.) (M. Lopes, Trad.). Porto Alegre: Artmed (Publicado originalmente em 1989)

Dahlblom, K., Herrara, A. R., Peña, R., \& Dahlgren, L. (2009). Home alone: children as caretakers in Leon, Nicaragua. Children \& Society, 23(1), 43-56.

De Toni, P. M., De Salvo, C. G., Marins, M. C., \& Weber, L. N. D. (2004). Etologia humana: um exemplo de apego. Psico, 9(1), 99-104.

Eibl-Eibesfeldt, I. (1989). Human ethology. New York: Aldine de Gruyter.

Fantz, R. L. (1963). Pattern vision in newborn infants. Science, 140(3564), 296-297.

Fisher, R. A. (1915). The evolution of sexual preference. Eugenics Review, 7, 184-192.

Frodi, A. M., Lamb, M. E., Leavitt, L. A., Donovan, W. L., Neff, C., \& Sherry, D. (1978). Fathers' and mothers' responses to the faces and cries of normal and premature infants. Developmental Psychology, 14(5), 490-498.

Fullard W., \& Reiling, A. M. (1976). An investigation of Lorenz's "babyness". Child Development, 47(4), 1191-1193.

Futuyma, D. J. (1992). Biologia evolutiva. Riberão Preto: Sociedade Brasileira de Genética/CNPq.

Gerald, M., Waitt, C., \& Maestripieri, D. (2006). An experimental examination of female responses to infant face coloration in rhesus macaques. Behavioural Processes, 73(3), 253-256.

Glocker, M. L., Langleben, D. D., Ruparel, K., Loughead, J. W., Gur, R. C., \& Sachser, N. (2009a). Baby schema in infant faces induces cuteness perception and motivation for caretaking in adults. Ethology, 115(3), 257-263.

Glocker, M., Langleben, D., Ruparel, K., Loughead, J., Valdez, J., Griffin, M., ... Gur, R. (2009b). Baby schema modulates the brain reward system in nulliparous women. Proceedings of the National Academy of Sciences of the United States of America, 106(22), 9115-9119.

Gould, S. J. (1989). O polegar do panda: reflexões sobre história natural. São Paulo: Martins Fontes.

Hafford, C. (no prelo). Sibling caretaking in immigrant families: understanding cultural practices to inform child welfare practice and evaluation. Evaluation and Program Planning.

Higley, J. D., Hopkins, W. D., Hirsch, R. M., Marra, L. M., \& Suomi, S. J. (1987). Preferences of female rhesus monkeys (Macaca mulatta) for infantile coloration. Developmental psychobiology, 20(1), 7-18.
Hildebrandt, K. A., \& Fitzgerald, H. E. (1979). Facial feature determinants of perceived infant attractiveness. Infant Behavior and Development, 2(4), 329-339.

Hildebrandt, K. A., \& Fitzgerald, H. E. (1983). The infant's physical attractiveness: its effect on bonding and attachment. Infant Mental Health Journal, 4(1), $1-12$.

Hinde, R. A., \& Barden, L. A. (1985). The evolution of the teddy bear. Animal Behaviour, 33(4), 1371-1373.

Hrdy, S. B. (2001). Mãe Natureza: uma visão feminina da evolução. Maternidade, filhos e seleção natural (A. Cabral, Trad.) Rio de Janeiro: Campus.

Izar, P. (2009). Ambiente de adaptação evolutiva. In E. Otta \& M. E. Yamamoto (Orgs.), Psicologia Evolucionista (pp. 22-32). São Paulo: Guanabara Koogan.

Konner, M. (1977). Evolution in human behavior development. In P. H. Leiderman, S. Tulkin, \& A. Rosenfeld (Orgs.), Culture and infancy: variations in human experience (pp. 69-109). New York: Academic Press.

Kosonen, M. (1996). Siblings as providers of support and care during middle childhood: children's perceptions. Children \& Society, 10(4), 267-279.

Krebs, E. A., \& Putland, D. A. (2004). Chic chicks the evolution of chick ornamentation in rails. Behavioral Ecology, 15(6), 946-951.

Kringelbach, M. L., Lehtonen, A., Squire, S., Harvey, A. G., Craske, M. G., Holliday, I. E., ... Stein, A. (2008). A specific and rapid neural signature for parental instinct. PLoS One, 3(2), e1664.

Langlois, H., Roggman, L. A., Casey, R. J., Ritter, J. M., Rieser-Danner, L. A., \& Jenkins, V.Y. (1987). Infant preferences for attractive faces: rudiments of a stereotype. Developmental Psychology, 23(3), 363-369.

Langlois, J. H., Ritter, J. M., Roggman, L. A., \& Vaughn, L. S. (1991). Facia diversity and infant preferences for attractive faces. Developmental Psychology, 27(1), 79-84.

Leakey, R. (1997). A origem da espécie humana. Rio de Janeiro: Rocco.

Lobmaier, J. S., Sprengelmeyer, R., Wiffen, B., \& Perrett, D. I. (2010). Female and male responses to cuteness, age and emotion in infant faces. Evolution and Human Behavior, 31(1), 16-21.

Lorenz, K. (1971). Studies in animal and human behavior (vol. II). London: Methuen.

Lorenz, K. (1995). Os fundamentos da etologia (P. M. Cruz, \& C. C. Alberts, Trad.). São Paulo: UNESP. (Publicado originalmente em 1981)

Lyon, B. E., Eadie, J. M., \& Hamilton, L. D. (1994). Paternal choice selects for ornamental plumage in American coot chicks. Nature, 371(6494), 240-243.

Maier, R. A., Holmes, D. L., Slaymaker, F. L., \& Reich, J. N. (1984). The perceived attractiveness of preterm infants. Infant Behavior \& Development, 7(4), 403-414.

Meltzoff, A. N., \& Moore, M. K. (1977). Imitation of facial and manual gestures by human neonates. Science, 198(4312), 75-78.

Mitani, J. C., \& Watts, D. (1997). The evolution of non-maternal caretaking among anthropoid primates: do helpers help? Behavioral Ecology and Sociobiology, 40(4), 213-220.

Mondloch, C. J., Lewis, T. L., Budreau, D. R., Maurer, D., Dannemiller, J. L., Stephens, B. R., \& Kleiner-Gathercoal, K. A. (1999). Face perception during early infancy. Psychological Science, 10(5), 419-422.

Morris, P. H., Reddy, V., \& Bunting, R. C. (1995). The survival of the cutest: who's responsible for the evolution of the teddy bear? Animal Behaviour, 50(6), 1697-1700.

Ochs, E., \& Schieffelin, B. (1984). Language acquisition and socialization. Three developmental stories and their implications. In R. Shweder, \& R. Le Vine (Orgs.), Culture theory (pp. 276-320). Cambridge: Cambridge University Press.

Poletto, M., Wagner, T. M. C., \& Koller, S. H. (2004). Resiliência e desenvolvimento infantil de crianças que cuidam de crianças: uma visão em perspectiva. Psicologia: Teoria e Pesquisa, 20(3), 241-250. 
Quinn, P. C., Kelly, D. J., Lee, K., Pascalis, O., \& Slater, A. M. (2008). Preference for attractive faces in human infants extends beyond conspecifics. Developmental Science, 11(1), 76-83.

Ritter, J. M., Casey, R. J., \& Langlois, J. H. (1991). Adults' responses to infants varying in appearance of age and attractiveness. Child Development, 62(1), 68-82.

Rosenberg, K., \& Trevathan, W. (1996). Bipedalism and human birth: the obstetrical dillema revised. Evolutionary Anthropology, 4(5), 161-168.

Sanefuji, W., Ohgami, H., \& Hashiya, K. (2007). Development of preference for baby faces across species in humans (Homo sapiens). Journal of Ethology, 25(3), 249-254.

Silk, J. (1999). Why are infants so attractive to others? The form and function of infant handling in bonnet macaques. Animal Behaviour, 57(5), 1021-1032,

Sprengelmeyer, R., Perrett, D. I., Fagan, E. C., Cornwell, R. E., Lobmaier, J. S., Sprengelmeyer, A., ... Young, A. W. (2009). The cutest little baby face: a hormonal link to sensitivity to cuteness in infant faces. Psychological
Science, 20(2), 149-154.

Tinbergen, N. (2005). On aims and methods of ethology. Animal Biology, 55(4), 297-321 (Publicado originalmente em 1963)

Treves, A. (1997). Primate natal coats: a preliminary analysis of distribution and function. American Journal of Physical Anthropology, 104(1), 47-70.

Vieira, M. L., \& Prado, A. B. (2004). Abordagem evolucionista sobre a relação entre filogênese e ontogênese no desenvolvimento infantil. In M. L. Seild de Moura (Org.), O bebê do século XXI e a psicologia em desenvolvimento (pp.155-199). São Paulo: Casa do Psicólogo.

West-Eberhard, M. J. (1983). Sexual Selection, Social Competition, and Speciation. The Quarterly Review of Biology, 58(2), 155-183.

Zebrowitz, L. A. (1997). Reading faces: Window to the soul? Boulder, Colorado: Westview Press.

Zukow-Goldrin, P. (1995). Sibling caregiving. In M. Bornstein (Org.), Handbook of parenting - Status and social conditions of parenting (Vol. 3) (pp. 177 208). Hillsdale, New Jersey: Erlbaum.

Monique Leitão, mestre em Psicobiologia pela Universidade Federal do Rio Grande do Norte, é professora de Psicologia do Instituto Federal de Educação, Ciência e Tecnologia do Rio Grande do Norte. Endereço para correspondência: Instituto Federal de Educação, Ciência e Tecnologia do Rio Grande do Norte. Av. Senador Salgado Filho, 1559, Tirol, Natal-RN, 59015-000. Telefone e Fax: (84) 4005-2639. Email: moniqueleitao@ yahoo.com.br

Rochele Castelo-Branco, mestre em Psicobiologia pela Universidade Federal do Rio Grande do Norte, é doutoranda em Psicobiologia da Universidade Federal do Rio Grande do Norte e bióloga da Empresa Brasileira de Infra-Estrutura Aeroportuária (INFRAERO). 